\title{
Characterizing Atypical BCL6 Signal Patterns Detected by Digital Fluorescence In Situ Hybridization (FISH) Analysis
}

Michael Liew (i), Ph.D. ${ }^{1}$, Leslie R. Rowe, M.S. ${ }^{1}$, Phillipe Szankasi, Ph.D. ${ }^{1}$, Christian N. Paxton, Ph.D. ${ }^{1}$, Todd Kelley, M.D. ${ }^{1,2}$, Reha M. Toydemir, M.D., Ph.D., ${ }^{1,2,}$, and Mohamed E. Salama, M.D., ${ }^{1,2}$

${ }^{1}$ ARUP Institute for Clinical and Experimental Pathology, Salt Lake City, UT, USA; Departments of ${ }^{2}$ Pathology and ${ }^{3}$ Pediatrics, University of Utah School of Medicine, Salt Lake City, UT, USA

Dear Editor,

The BCL6 gene encodes a 706-amino acid sequence-specific repressor transcription factor [1]. BCL6 has been identified as commonly rearranged in 20-40\% of diffuse large B cell lymphomas (DLBCL) [2, 3]. BCL6 can be rearranged with the immunoglobulin (Ig) gene loci or non-lg gene partners [2]. Breakapart (BAP) FISH probes are widely used for clinical testing of genes with multiple translocation partners [4]. The expected signal pattern of a rearranged sample using a BAP FISH probe is a single red and single green signal for derivative chromosomes and a single fusion signal for the normal homolog (1F/1R/1G). However, because of the complexity of genomic changes that can occur in cancer, signal patterns other than $1 F / 1 R / 1 G$, that is, atypical or unusual signal patterns, are also observed [5]. We describe the validation of a locus specific identifier (LSI)-BCL6 FISH assay using a digital analysis system, as well as our experience with unusual signal patterns observed using this assay. We demonstrate that unusual FISH signal patterns can be associated with copy number alterations in addition to rearrangements involving the BCL6 locus at 3q27. This study was granted exempt status by the University of Utah Institutional Review Board
(IRB_00084247).

We evaluated 34 formalin-fixed, paraffin-embedded tissue samples to validate the LSI-BCL6 FISH probe. The procedures for preparing, scanning, and analyzing the hematoxylin and eosin (H\&E) and FISH slides traditionally and digitally have been described previously [6]. FISH cutoffs for the total rearranged nuclei were calculated using the correlation curve from regression analysis of traditional versus digital FISH. Samples with $\geq 24 \%$ total rearranged nuclei were considered positive for $B C L 6$ rearrangement; samples with $\leq 15 \%$ total rearranged nuclei were considered negative for $B C L 6$ rearrangement. Those between $16 \%$ and $23 \%$ were interpreted at the discretion of the attending pathologist. If a sample appeared to have an elevated number of unusual BCL6 signal patterns, it was considered positive for BCL6 rearrangement. Four additional samples were identified as having unusual signal patterns after completion of the validation. Two of these had sufficient sample left to perform additional molecular testing. The OncoScan genome-wide molecular inversion probe (MIP) array (ThermoFisher Scientific, Waltham, MA, USA), which contains over $220 \mathrm{~K}$ probes, was used to identify chromosomal gains or losses, as previously de-
Received: October 9, 2017

Revision received: March 2, 2018

Accepted: June 21, 2018

Corresponding author: Michael Liew

(iD https://orcid.org/0000-0003-0783-545X

ARUP Institute for Clinical and Experimental Pathology, 500 Chipeta Way,

Salt Lake City, UT 84108-1221, USA

Tel: +1-801-583-2787 ext. 2179, Fax: +1-801-584-5207

E-mail: liewm@aruplab.com

\section{(c) Korean Society for Laboratory Medicine}

This is an Open Access article distributed under the terms of the Creative Commons Attribution Non-Commercial License (http://creativecommons.org/licenses/by-nc/4.0) which permits unrestricted non-commercial use, distribution, and reproduction in any medium, provided the original work is properly cited. 
scribed [7]. Next generation sequencing (NGS) was used to determine the sequence of the 3q27 loci.

Regression analysis showed a strong correlation $\left(R^{2}=0.9\right)$ be-

Table 1. Distribution of unusual signal patterns in samples tested for BCL6 rearrangement

\begin{tabular}{lrrrc}
\hline Unusual signal patterns* & $1 \mathrm{~F} / 1 \mathrm{G}$ & $1 \mathrm{~F} / 1 \mathrm{R}$ & $2 \mathrm{~F} / 1 \mathrm{G}$ & $2 \mathrm{~F} / 1 \mathrm{R}$ \\
\hline Sample 1U & $18.5^{\dagger}$ & 2.9 & 14.9 & 0.3 \\
Sample 2U & 2.2 & 10.6 & 0.4 & 9.2 \\
Sample 3U & 7.3 & 1.0 & 1.0 & 0.5 \\
Sample 4U & 2.2 & 27.6 & 0.2 & 26.7 \\
Average & 7.6 & 10.5 & 4.1 & 9.2 \\
SD & 7.7 & 12.1 & 7.2 & 12.4 \\
Negative & & & & \\
Average & 1.6 & 1.2 & 1.1 & 0.8 \\
SD & 1.5 & 1.1 & 1.1 & 1.0 \\
Positive & & & & \\
Average & 9.9 & 11.1 & 2.9 & 1.8 \\
SD & 8.6 & 6.9 & 1.6 & 2.2 \\
\hline
\end{tabular}

${ }^{*}$ The distribution of unusual signal patterns in the four unusual samples is shown and compared with the average distribution in the 3q27-negative and -positive groups. The averages were derived from the validation samples (12 positive and 22 negative 3q27-rearranged samples).

†Values are expressed as a percentage of the total number of countable signal patterns identified in a sample.

Abbreviations: F, fused signal; $G$, green signal; $R$, red signal. tween the LSI-BCL6 assay by traditional and GenASIs (digital) FISH (Applied Spectral Imaging, Carlsbad, CA, USA). The digital FISH system accurately classified 12/12 BCL6 rearrangementpositive samples (100\%) and 22/22 BCL6 rearrangement-negative samples (100\%). Unusual signal patterns were defined as $\mathrm{nF} / \mathrm{nR}$ and $\mathrm{nF} / \mathrm{nG}$ (Table 1).

Sample $1 \mathrm{U}$, which had elevated $1 \mathrm{~F} / 1 \mathrm{G}$ and $2 \mathrm{~F} / 1 \mathrm{G}$ FISH signal patterns, was found to have a copy number gain by MIP array and a rearrangement by NGS (Fig. 1). Sample $3 U$, which had elevated levels of $1 F / 1 G$, was shown to have a BCL6 rearrangement by NGS. NGS identified the BCL6 translocation partner in sample $1 \mathrm{U}$ and $3 \mathrm{U}$ as $I G H$ and interleukin receptor 21 (IL21R), respectively.

The strong correlation found between traditional and digital FISH analysis methods for BCL6 rearrangements using the LSIBCL6 fusion probe is similar to our previous study [6]. Research has identified unusual signal patterns using BAP FISH probes. Vargas et al [5] described atypical/unusual signal patterns identified using an EWSR1 BAP probe, as well as issues concerning their identification. We could identify losses of both the $3^{\prime}$ and 5 ends of the BCL6 gene. Despite their low frequency (2\%), trying to determine how to interpret these unusual signal patterns was important.

NGS confirmed BCL6 translocations in samples $1 \mathrm{U}$ and $3 \mathrm{U}$. Sample $1 \mathrm{U}$ had elevated FISH signal patterns, suggesting a de-

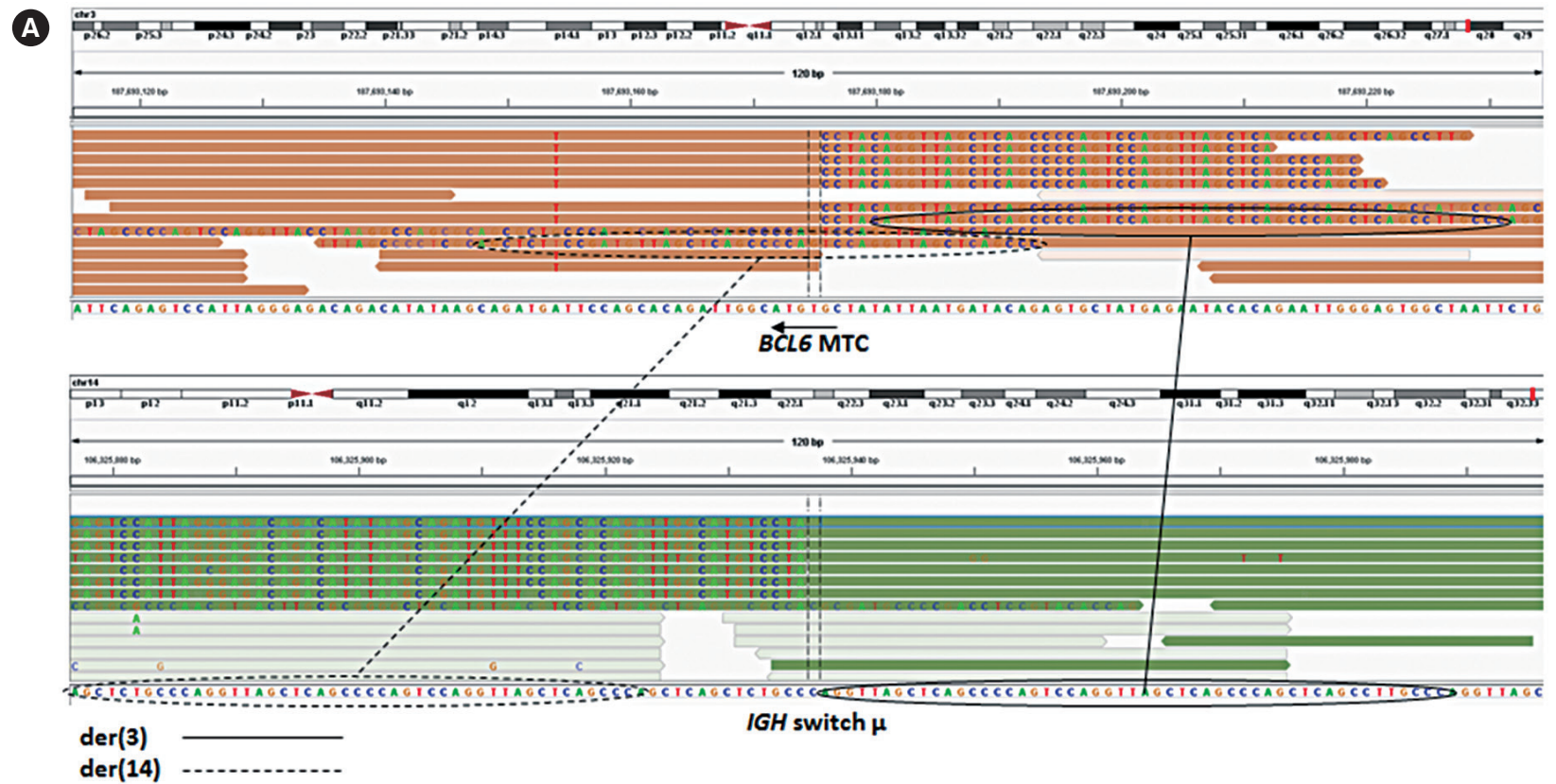

Fig. 1. Results from the combined testing of sample $1 U$. (A) Next generation sequencing identified a BCL6-IGH fusion.

(Continued to the next page) 


\section{B}

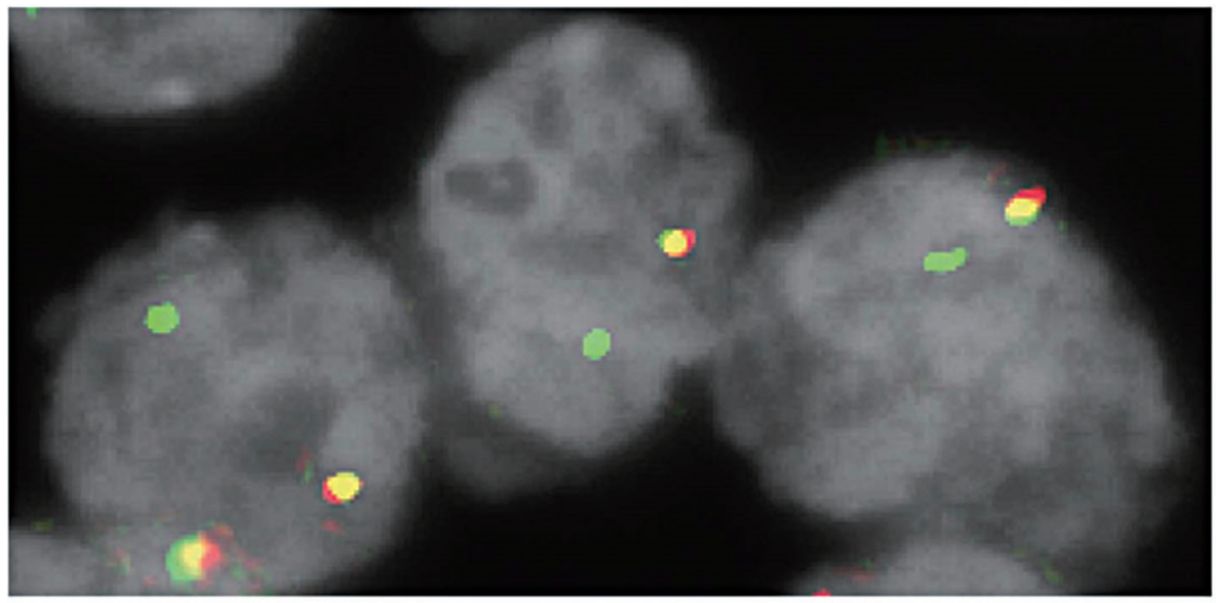

(
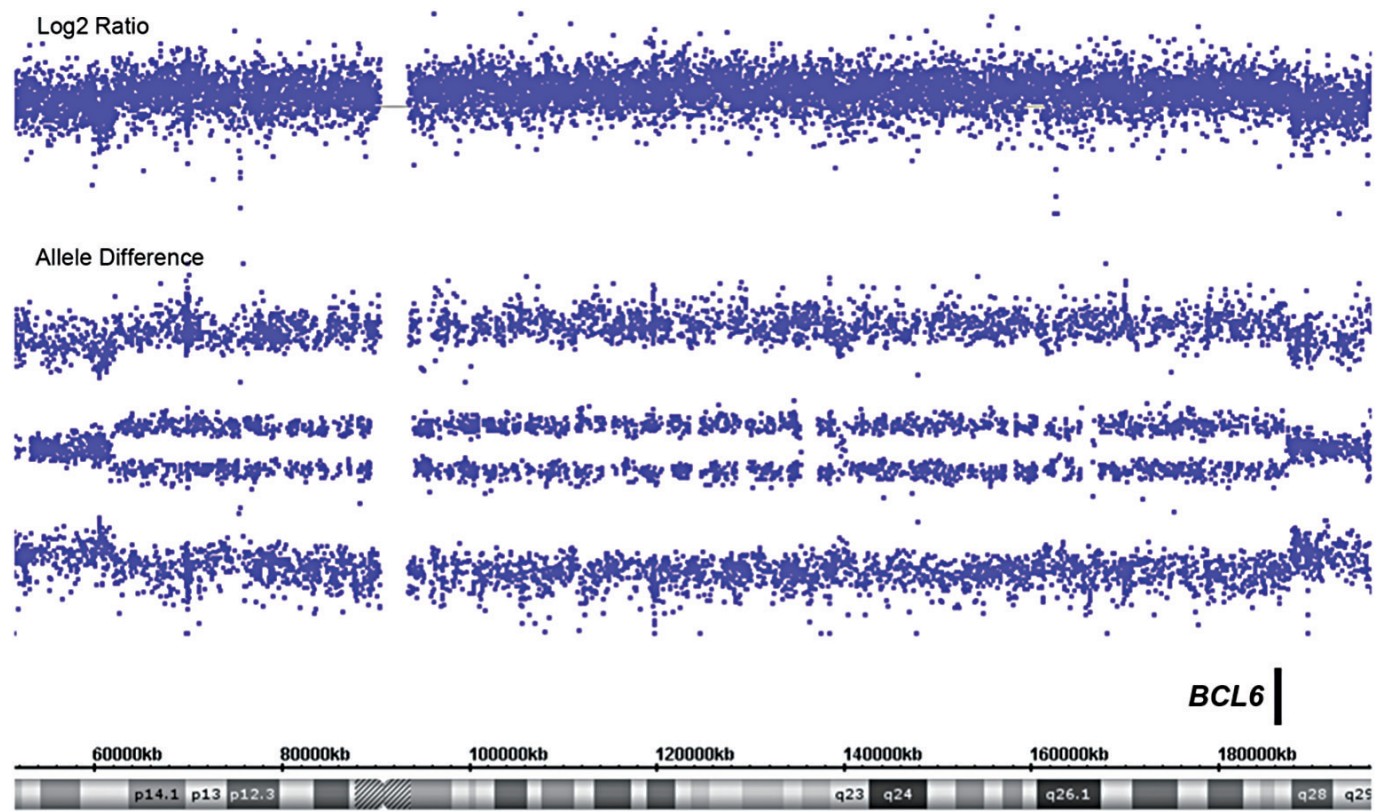

Fig. 1. Continued. (B) Representative cells with $1 F / 1 G$ and $2 F / 1 G$ signal patterns with the BCL6 probe set. (C) The molecular inversion probe array analysis detected a copy number gain from 3p14.2 to 3q27.3.

letion; however, the MIP array indicated a gain. This probably reflects clonal heterogeneity as FISH results correspond to individual cells, whereas the MIP array result is based on DNA extracted from all cells within the tissue. Identification of the causative genetic mechanism may not always be possible; however, as unusual FISH signal patterns are often associated with a copy number gain and rearrangement, combined utilization of FISH, NGS, and microarray technologies will be helpful in most cases. Our findings highlight the importance of follow-up investigation by NGS and microarray for abnormal signal patterns in initial FISH screening for better clinical management.

\section{Authors' Disclosures of Potential Conflicts of Interest}

No potential conflicts of interest relevant to this article are reported.

\section{Acknowledgment}

This work was funded by the Institute for Clinical and Experimental Pathology, ARUP Laboratories. The authors would like to thank Kristina Moore for her technical assistance with the optimization and implementation of the LSI-BCL6 digital FISH scanning. 


\section{REFERENCES}

1. Albagli-Curiel O. Ambivalent role of BCL6 in cell survival and transformation. Oncogene 2003;22:507-16.

2. Ohno $\mathrm{H}$ and Fukuhara S. Significance of rearrangement of the BCL6 gene in B-cell lymphoid neoplasms. Leuk Lymphoma 1997;27:53-63.

3. Willis TG and Dyer MJ. The role of immunoglobulin translocations in the pathogenesis of B-cell malignancies. Blood 2000;96:808-22.

4. Martin-Subero JI, Gesk S, Harder L, Grote W, Siebert R. Interphase cytogenetics of hematological neoplasms under the perspective of the novel WHO classification. Anticancer Res 2003;23:1139-48.
5. Vargas AC, Selinger CI, Satgunaseelan L, Cooper WA, Gupta R, Stalley P, et al. Atypical Ewing sarcoma breakpoint region 1 fluorescence in-situ hybridization signal patterns in bone and soft tissue tumours: diagnostic experience with 135 cases. Histopathology 2016;69:1000-11.

6. Liew M, Rowe L, Clement PW, Miles RR, Salama ME. Validation of break-apart and fusion MYC probes using a digital fluorescence in situ hybridization capture and imaging system. J Pathol Inform 2016;7:20.

7. Paxton CN, Rowe LR, South ST. Streamlining the OncoScan array procedure for use in a clinical laboratory. J Assoc Genet Technol 2015;41: 61-5. 